\title{
Association between Neutrophil to Lymphocyte Ratio and Severity of Coronary Artery Disease in Chronic Stable Angina
}

\author{
Ratan Kumar Datta, Md. Mamunur Rashid, MG Azam, Md. Salahuddin Ulubbi, \\ Mohammad Khalilur Rahman Siddiqui, Pranob Karmaker, Morshedul Ahsan, Jatindra Nath Saha, \\ Muhammad Azmol Hossain, Abul Hasnat Md. Jafar \\ Department of Cardiology, National Institute of Cardiovascular Diseases, Dhaka
}

\begin{abstract}
Key words:

Background: Neutrophil to lymphocyte ratio (NLR) has been proposed as a new prognostic marker in Neutrophil , lymphocyte, Ischaemic heart disease, Coronary artery. patients with chronic stable angina (CSA). NLR is a cheap, easily available, non-invasive and routinely done procedure to predict the severity of coronary artery disease.

Methods: 110 patients with stable coronary artery disease were evaluated to calculate the NLR from January, 2016 to September, 2016. The patients were divided into two groups: Group I NLR>2.38 and group II NLRd"2.38. Coronary angiogram was done during index hospitalization. The severity of the coronary artery disease was assessed by vessel score and Gensini score and was compared between the groups.

Results: NLR was significantly higher in the group of high vessel score and high Gensini score. We found significant weak association between NLR and vessel score $(r=0.30, p=0.004)$ and a moderate positive correlation between NLR and Gensini score $(r=0.65, P=0.001)$. With the increase of NLR, vessel score and Gensini score increases demonstrating more severe coronary artery disease. Univariate logistic regression analysis of variables of interest revealed that age, hypertension, dyslipidemia, serum creatinine, total WBC count and NLR were independent predictor of severe coronary artery disease with odds ratio (OR) being $1.88,3.93,5.01,5.54,4.05$ and 5.70 respectively. In multivariate logistic regression analysis, after adjustment of factors NLR remain independent predictors of severe CSA $(O R=5.73 ; p=0.002)$.

Conclusion: Increased neutrophil to lymphocyte ratio is associated with angiographically severe coronary artery disease in chronic stable angina and this association is independent of conventional cardiovascular risk factors.
\end{abstract}

(Cardiovasc. j. 2018; 10(2): 164-170)

\section{Introduction:}

Chronic stable angina is generally characterized by episodes of reversible myocardial demand/ supply mismatch, related to ischemia or hypoxia, which are usually inducible by exercise, emotion or other stress and reproducible-but, which may also be occurring spontaneously. The various clinical presentations of CSA are associated with different underlying mechanisms that mainly include: (i) plaque-related obstruction of epicardial arteries; (ii) focal or diffuse spasm of normal or plaque-diseased arteries; (iii) microvascular dysfunction and (iv) left ventricular dysfunction caused by prior acute myocardial necrosis and/or hibernation (ischemic cardiomyopathy). ${ }^{1}$
The role of inflammatory markers in cardiovascular diseases has been studied extensively and a consistent relationship between various inflammatory markers and cardiovascular diseases has been established in the past. Among these C-reactive protein (CRP), high sensitive C-reactive protein (hs-CRP), fibrinogen, interleukin-6 (IL-6), monocyte/ macrophage colony stimulating factor (MCSF), tumor necrosis factor-alpha, lipoprotein associated phospholipase-A2 and interleukin-1 isoform are noteworthy. Recently, neutrophil to lymphocyte ratio has emerged as a new addition to the long list of this inflammatory markers. ${ }^{2}$ A higher NLR indicates a higher level of inflammation and integrates the predictive

Address of correspondence: Dr. Ratan Kumar Datta, Department of Cardiology, National Institute of Cardiovascular Diseases, Dhaka, Bangladesh. Email- ratandattamd@gmail.com 
risk of these two leukocyte subtypes into a single risk factor. ${ }^{3}$

NLR is a combination of two independent markers of inflammation: neutrophils as a marker of ongoing nonspecific inflammation and lymphocytes as a marker of the regulatory pathway. ${ }^{4}$ NLR is calculated by dividing the number of neutrophils by the number of lymphocytes, from peripheral blood sample. Previous study has shown that if the NLR is higher there is increased severity of CAD and higher is the Gensini scores. Coronary angiography is the gold standard for the clinical judgment of CAD, whereas the Gensini score is a quantitative indicator for the estimation of the severity of coronary artery stenosis on the basis of coronary angiography. ${ }^{5}$

\section{Methods:}

This cross sectional study was conducted in the Department of Cardiology, National Institute of Cardiovascular Diseases, Dhaka, from December 2015 to November 2016. Patients with chronic stable angina who got admitted for coronary angiogram during the study period were selected by purposive sampling. Patients who underwent prior PCI and/or CABG, patients with heart failure - NYHA class III, IV, hematological diseases, malignancy, chronic kidney disease, chronic liver disease, ongoing infection, chronic inflammatory disease, autoimmune disease were excluded from the study. Total 110 cases were included in the study and were divided into two groups on the basis of NLR cut off level 2.8: Group I (NLR > 2.38) and Group II (NLR d" 2.38). After taking informed written consent from each patient meticulous history was taken and detailed clinical examination was performed and recorded in predesigned structured proforma. Levels of hemoglobin, white blood cells, neutrophils, lymphocytes, other differentials of white blood cells and platelets were determined by automated hematology analyzer. Serum creatinine, random blood sugar, fasting lipid profile and other screening tests for coronary angiogram were done. Coronary angiogram was done by conventional method in the same hospital setting. Severity assessment was done by Gensini score and vessel score.

NLR was calculated by dividing the number of neutrophils by the number of lymphocytes, from peripheral blood sample. Angiographic pattern and severity of coronary artery disease were assessed by interpretation of coronary angiogram by visual estimation by two cardiologists. Severity of coronary stenosis was graded according to the number of major epicardial vessel with significant stenosis (vessel score) and Gensini score.

In vessel score, significant coronary artery disease was defined as $>70 \%$ stenosis in any of the three major epicardial coronary arteries or a left main coronary artery stenosis $>50 \%$. Angiograms revealing coronary artery stenosis $<70 \%$ in major epicardial coronary arteries were termed non-obstructive CAD. Extent of coronary artery disease was defined as significant single, two or three vessel coronary artery disease. Score ranged from 0 to 3 depending on the number of vessel involve. Left main coronary artery was scored as single vessel diseasei) Score $0=$ no vessel involvement, ii) Score $1=$ single vessel involvement, iii) Score $2=$ double vessel involvement, iv) Score $3=$ triple vessel involvement.

The Gensini score was developed by Gensini and takes into consideration the geometrical severity of lesions by angiography, the cumulative effects of multiple obstructions, and the significance of jeopardized myocardium. A nonlinear score was assigned to each lesion based on the severity of stenosis as indicated by the reduction of lumen diameter. A multiplier was applied to each lesion score based upon its location in the coronary tree depending on the functional significance of the area supplied by that segment. The final Gensini score was the sum of the lesion scores. The score assessed 14 coronary artery segments, which were scored according to their anatomical importance (ranging from 0.5 to 5 ) multiplied by the score regarding the maximum degree of obstruction. The points of the 14 segments were summed up to yield a final score. 
Total Gensini Score was calculated as:

\begin{tabular}{lc}
\hline$\%$ of stenosis & Score \\
\hline $1-25 \%$ & 1 \\
$26-50 \%$ & 2 \\
$51-75 \%$ & 4 \\
$76-90 \%$ & 8 \\
$91-99 \%$ & 16 \\
$100 \%$ & 32 \\
\hline
\end{tabular}

\begin{tabular}{lc}
\hline Vessel (S) involved & Score \\
\hline Left Main & 5 \\
Proximal LAD /Proximal LCX & 2.5 \\
Mid LAD & 1.5 \\
Distal LAD/ Distal LCX/D1/ OM/ Prox RCA/ & 1 \\
Mid RCA/Distal RCA /PDA/PLV & \\
D2 & 0.5 \\
\hline
\end{tabular}

Total Gensini score $=$ Sum of (Score for \% of stenosis X Score for Vessel(s) involved)

Interpretation of coronary angiogram was made as the Gensini score. According to Gensini Score CAD was categorized as mild (1-10), moderate (10-50) and severe (>50).

The SPSS Statistical Software (16.0 version, SPSS Inc., Chicago, Illinois, USA) was used for data analysis. Continuous variables were expressed in mean \& standard deviation and categorical variables as frequency and percentage. Quantitative variables were analyzed by Student's t-test and Categorical variables were analyzed by chi-square test and Fisher's exact test as applicable. To test association between NLR and coronary artery disease severity Pearson's and Spearman's rank order correlation test were used. Univariate and multivariate logistic regression analysis were done to evaluate the independent predictors of severity of CAD. The odds ratio (OR) and 95\% confidence interval were calculated. $p$ value of less than 0.05 was considered as significant.

\section{Results:}

Among the total 110 patients group-I and groupII had 55 patients each. The mean age difference between the group was statistically significant $(p=0.001)$. Male: Female ratio was 10:1. Among the conventional CVD risk factors, hypertension and dyslipidemia were significantly high in group-É $(p<0.05)$. No significant difference $(p>0.05)$ between two groups was found in case of diabetes mellitus, smoking and family history of CAD (Table I).

The differences in mean hemoglobin and RBS levels between two groups were insignificant $(p>0.05)$. But the differences in serum creatinine levels was significant $(\mathrm{p}<0.05)$. In lipid profile study, only HDL was significantly low in group-I $(p<0.01)$. Patients of group-I showed significantly higher mean WBC counts $(\mathrm{p}<0.01)$. Mean count of neutrophil and lymphocyte were statistically significant $(\mathrm{p}<0.05)$ across the group. The Mean NLR was $3.18 \pm 0.79$ in group-E \& $1.96 \pm 0.42$ in group-ÉÉ and the difference was statistically significant $(p=0.001)$. Mean LVEF also showed statistically significant difference between two groups $(\mathrm{p}<0.05)$ (Table II).

This study found that in "vessel score 0" and "vessel score 2" categories there was significant difference $(p<0.05)$ between the groups and it was low in group-I, whereas the difference between groups in "vessel score 3" category was significantly high $(p=0.001)$ in the same group (Table III). Gensini scoring found that mild CAD was significantly low in group É $(p<0.05)$. Moderate CAD showed insignificant difference $(p>0.05)$ between the groups. Severe CAD was significantly higher in group É $(\mathrm{p}=0.001)$ (Table IV).

There was a positive correlation between NLR and coronary artery disease severity in terms of vessel score $(r=0.30)$. It was observed statistically significant ( $\mathrm{p}=0.004)$ by Spearman's rank order correlation test (figure 1). There was also a positive correlation between NLR and coronary artery disease severity in terms of Gensini score $(r=0.65)$. With the increase of NLR Gensini score increases. It was found statistically significant ( $\mathrm{p}=0.001)$ by Pearson's correlation test (Figure 2).

Univariate logistic regression analysis of variables showed that age $>50$ years, hypertension, dyslipidemia, serum creatinine, WBC count and NLR were the significant predictors of severe CAD with ORs being 1.88, $3.93,5.01,5.54,4.05$ and 5.70 respectively (Table V). In multivariate logistic regression analysis age $>50$ years, hypertension, dyslipidemia and NLR were found the independent predictors of severe CAD with ORs being 3.27, 3.71, 4.95 and 5.73 respectively (Table VI). 
Table-I

Demographic and clinical characteristics of study patients $(N=110)$.

\begin{tabular}{lccc}
\hline Variables & Group I $(\mathrm{n}=55)$ & Group II $(\mathrm{n}=55)$ & $\mathrm{p}$ value \\
\hline Age, mean \pm SD, years & $57.4 \pm 8.7$ & $51.6 \pm 8.2$ & $0.001^{\mathrm{s}}$ \\
Sex, male: female & $10: 1$ & $10: 1$ & $1.0^{\mathrm{ns}}$ \\
Hypertension, n (\%) & $25(45.5)$ & $14(25.5)$ & $0.03^{\mathrm{s}}$ \\
Diabetes mellitus, n (\%) & $10(18.2)$ & $7(13)$ & $0.56^{\mathrm{ns}}$ \\
Dyslipidaemia, n (\%) & $19(34.5)$ & $9(16.4)$ & $0.02^{\mathrm{s}}$ \\
Smoking, n (\%) & $18(32.7)$ & $16(29.1)$ & $0.68^{\mathrm{ns}}$ \\
Family history of CAD, n (\%) & $6(10.9)$ & $4(7.3)$ & $0.47^{\mathrm{ns}}$ \\
\hline
\end{tabular}

Group I = NLR $>2.38$

Group II = NLR d" 2.38

$\mathrm{s}=$ significant

$\mathrm{ns}=$ not significant

$\mathrm{p}$ value reached from unpaired t-test.

Table-II

Laboratory characteristics of study patients $(N=110)$

\begin{tabular}{lccc}
\hline Variables & Group I $(\mathrm{n}=55)$ & Group II $(\mathrm{n}=55)$ & p value \\
\hline Hb, mean $\pm \mathrm{SD},(\mathrm{gm} / \mathrm{dl})$ & $12.6 \pm 1.1$ & $12.9 \pm 1.20$ & $0.64^{\mathrm{ns}}$ \\
WBC count $(\mathrm{K} / \mu \mathrm{L})$ & $9.4 \pm 2.6$ & $7.5 \pm 1.90$ & $0.006^{\mathrm{s}}$ \\
Neutrophil count $(\mathrm{K} / \mu \mathrm{L})$ & $7.5 \pm 1.0$ & $5.1 \pm 0.9$ & $0.04^{\mathrm{s}}$ \\
Lymphocyte count $(\mathrm{K} / \mu \mathrm{L})$ & $2.4 \pm 0.6$ & $3.1 \pm 0.7$ & $0.03^{\mathrm{s}}$ \\
NLR & $3.18 \pm 0.79$ & $1.96 \pm 0.42$ & $0.001^{\mathrm{s}}$ \\
RBS (mmol/lit) & $8.8 \pm 3.1$ & $8.3 \pm 2.9$ & $0.57^{\mathrm{ns}}$ \\
Lipid profile $(\mathrm{mg} / \mathrm{dl})$ & & & \\
Total cholesterol & $171.10 \pm 13.30$ & $166.10 \pm 12.50$ & $0.08^{\mathrm{ns}}$ \\
$\quad$ LDL & $120.60 \pm 12.90$ & $114.50 \pm 15.20$ & $0.09^{\mathrm{ns}}$ \\
$\quad$ HDL & $35.50 \pm 7.20$ & $39.8 \pm 7.60$ & $0.003^{\mathrm{s}}$ \\
$\quad$ S. Triglyceride & $132.60 \pm 17.80$ & $127.70 \pm 16.90$ & $0.17^{\mathrm{ns}}$ \\
Serum creatinine $(\mathrm{mg} / \mathrm{dl})$ & $1.3 \pm 0.20$ & $0.80 \pm 0.20$ & $0.02^{\mathrm{s}}$ \\
LVEF, mean $\pm \mathrm{SD},(\%)$ & $49.60 \pm 7.30$ & $54.30 \pm 7.10$ & $0.02^{\mathrm{s}}$ \\
\hline
\end{tabular}

Group I $=$ NLR $>2.38$

Group II $=$ NLR $\leq 2.38$

$\mathrm{s}=$ significant

$\mathrm{ns}=$ not significant

$\mathrm{p}$ value reached from unpaired t-test

Table-III

Distribution of the study patients according to vessel score $(N=110)$.

\begin{tabular}{lcccccc}
\hline Severity of CAD & \multicolumn{2}{c}{ Group I $(\mathrm{n}=55)$} & & \multicolumn{2}{c}{ Group II $(\mathrm{n}=55)$} & $\mathrm{p}$ value \\
\cline { 2 - 3 } (Vessel score) & Number & $\%$ & & Number & $\%$ & \\
\hline Score 0 & 4 & 7.3 & & 8 & 14.5 & $0.02^{\mathrm{s}}$ \\
Score 1 & 22 & 40 & & 25 & 45.6 & $0.40^{\mathrm{ns}}$ \\
Score 2 & 13 & 23.6 & & 21 & 38.2 & $0.03^{\mathrm{s}}$ \\
Score 3 & 16 & 29.1 & & 01 & 1.8 & $0.001^{\mathrm{s}}$ \\
\hline
\end{tabular}

Group I $=$ NLR $>2.38$

Group II = NLR d" 2.38

$\mathrm{s}=$ significant

$\mathrm{ns}=$ not significant

$\mathrm{p}$ value reached from Chi Square test 
Table-IV

Distribution of the study patients according to Gensini score $(N=110)$.

\begin{tabular}{lcccccc}
\hline Severity of CAD & \multicolumn{2}{c}{ Group I $(\mathrm{n}=55)$} & & \multicolumn{2}{c}{ Group II $(\mathrm{n}=55)$} & $\mathrm{p}$ value \\
\cline { 2 - 4 } (Gensini score) & Number & $\%$ & & Number & $\%$ & \\
\hline Mild $(1-10)$ & 5 & 9.1 & & 13 & 23.6 & $0.04^{\mathrm{s}}$ \\
Moderate $(10-50)$ & 33 & 60.0 & & 38 & 69.1 & $0.31^{\text {ns }}$ \\
Severe $(>50)$ & 17 & 30.9 & 4 & 7.3 & $0.001^{\mathrm{s}}$ \\
\hline
\end{tabular}

Group I $=$ NLR $>2.38$

Group II = NLR d” 2.38

$\mathrm{s}=$ significant

$\mathrm{ns}=$ not significant

$\mathrm{p}$ value reached from Chi Square test

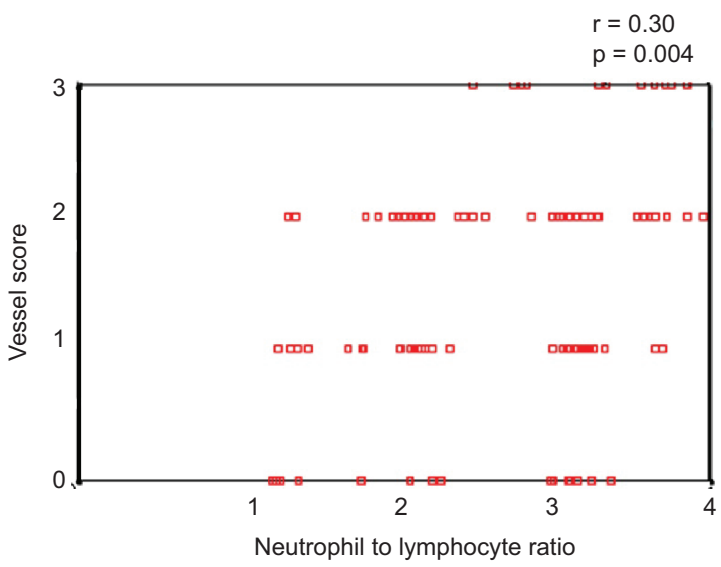

Fig 1: Correlation between NLR and vessel score by Spearman's rank order correlation test.

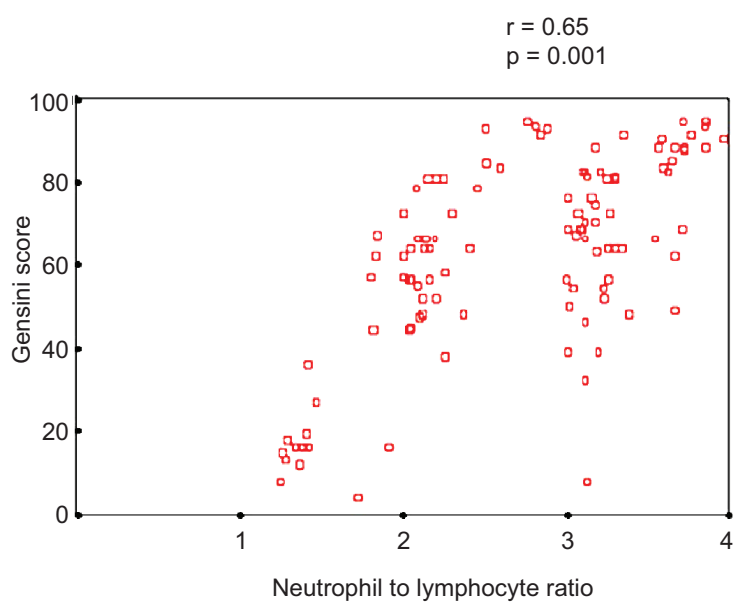

Fig 2: Correlation between NLR and Gensini score by Pearson's correlation test.

Table-V

Univariate logistic regression analysis of determinants of severe CAD.

\begin{tabular}{lccccc}
\hline Variables of interest & $\hat{\mathrm{a}}$ & $\mathrm{S} . \mathrm{E}$. & $\mathrm{p}$ value & OR & $95 \%$ CI \\
\hline Age> 50 years & 0.534 & 0.557 & $0.03^{\mathrm{s}}$ & 1.88 & $0.632-2.662$ \\
Hypertension & 1.425 & 0.399 & $0.007^{\mathrm{s}}$ & 3.93 & $1.233-9.781$ \\
Smoking & 0.138 & 0.517 & 0.38 & 1.14 & $0.417-3.164$ \\
Family history of premature CAD & 0.127 & 0.327 & 0.44 & 0.74 & $0.053-3.715$ \\
Diabetes mellitus & 0.371 & 0.506 & 0.107 & 0.93 & $0.460-1.620$ \\
Dyslipidemia & 1.867 & 0.531 & $0.004^{\mathrm{s}}$ & 5.01 & $2.831-11.704$ \\
Serum creatinine & 2.001 & 0.603 & $0.04^{\mathrm{s}}$ & 5.54 & $2.934-12.111$ \\
WBC count $(\mathrm{K} / \mu \mathrm{L})$ & 1.225 & 0.349 & $0.006^{\mathrm{s}}$ & 4.05 & $1.332-9.285$ \\
Neutrophil count $(\mathrm{K} / \mu \mathrm{L})$ & 0.234 & 0.139 & 0.09 & 1.11 & $0.349-1.201$ \\
Lymphocyte count $(\mathrm{K} / \mu \mathrm{L})$ & 0.201 & 0.101 & 0.14 & 0.96 & $0.789-1.001$ \\
LVEF & 0.213 & 0.104 & 0.12 & 0.95 & $0.888-1.015$ \\
NLR & 1.741 & 0.596 & $0.003^{\mathrm{s}}$ & 5.70 & $1.775-18.330$ \\
\hline
\end{tabular}

Dependent variable: Severe CAD (Gensini score $>50)$

Independent variables: Age >50 years, Hypertension, Smoking, Family H/O of premature CAD, Diabetes mellitus, Dyslipidemia, Serum creatinine, WBC count, Neutrophil count, Lymphocyte count, LVEF and NLR.

$\mathrm{s}=$ Significant 
Table-VI

Multivariate logistic regression analysis of determinants of severe CAD.

\begin{tabular}{lccccc}
\hline Variables of interest & $\beta$ & S.E. & p value & OR & $95 \%$ CI \\
\hline Age $>50$ & 1.434 & 0.392 & $0.02^{\mathrm{s}}$ & 3.27 & $1.219-1.658$ \\
Hypertension & 1.245 & 0.499 & $0.006^{\mathrm{s}}$ & 3.71 & $1.234-9.789$ \\
Dyslipidemia & 1.629 & 0.512 & $0.003^{\mathrm{s}}$ & 4.95 & $2.110-10.111$ \\
Neutrophil count $(\mathrm{K} / \mu \mathrm{L})$ & 0.234 & 0.139 & 0.09 & 1.11 & $0.349-1.201$ \\
Lymphocyte count $(\mathrm{K} / \mu \mathrm{L})$ & 0.201 & 0.101 & 0.14 & 0.96 & $0.789-1.001$ \\
NLR & 1.729 & 0.582 & $0.002^{\mathrm{s}}$ & 5.73 & $1.725-17.234$ \\
\hline
\end{tabular}

Dependent variable: Severe CAD (Gensini score >50)

Independent variables: Age >50 years, Hypertension, Dyslipidemia, Neutrophil count, Lymphocyte count and NLR. $\mathrm{s}=$ Significant

\section{Discussion:}

The mean age of the studied patients was a $54.7 \pm 11.4$ year ranging from 43 to 66 years. The mean age of group I patients was significantly $(\mathrm{p}=0.001)$ higher than group II. In a similar study conducted by Zhang, et al. mean age was $66 \pm 10$ years in group with NLR $>2.38$ (high Gensini score group) and $61 \pm 10$ years in group with NLRd" 2.38 and difference was statistically significant $(p=0.001){ }^{6}$ It was evident from the study that group-I tended to be older than group-II.

In group-I the mean WBC count was $9.4 \pm 2.6$ (K/ $\mu \mathrm{L}$ ) and in group-II mean WBC count was $7.5 \pm 1.90(\mathrm{~K} / \mu \mathrm{L})$ and this difference was statistically significant. In a similar study conducted by Kaya et al. found mean WBC count $9.9 \pm 3.1(\mathrm{~K} / \mu \mathrm{L})$ in higher NLR group and $8.3 \pm 2.8$ $(\mathrm{K} / \mu \mathrm{L})$ in lower NLR group and the difference was statistically significant $(\mathrm{p}<.001) .{ }^{7}$ Mean neutrophil count was $7.5 \pm 1.0$ and $5.1 \pm 0.9(\mathrm{~K} / \mu \mathrm{L})$, mean lymphocyte count was $2.4 \pm 0.6$ and $3.1 \pm 0.7$ $(\mathrm{K} / \mu \mathrm{L})$ and mean NLR was $3.18 \pm 0.79$ and $1.96 \pm 0.42$ in group-I and group-II respectively and the differences in the values between the two groups were statistically significant $(\mathrm{p}<0.05)$. In a similar study done by Zhang, et al. found mean neutrophil count was $6.6+1.0$ and $6.1+1.0$ $(\mathrm{K} / \mu \mathrm{L})$, mean lymphocyte count was $2.9 \pm 0.9$ and $2.4 \pm 0.8(\mathrm{~K} / \mu \mathrm{L})$ and mean NLR was $3.4 \pm 2$ and $2.5 \pm 1.6$ in high and low NLR group respectively. ${ }^{6}$

Mean left ventricular ejection fraction (EF\%) was $49.6 \pm 7.3 \%$ in Group I and $53.3 \pm 8.5 \%$ in Group II. The difference of mean between the two groups was statistically significant $(p=0.02)$. In a study $\mathrm{Na}$, et al. found mean $\mathrm{EF} \% 58.0 \pm 10.5 \%$ in severe coronary artery disease group and $59.6 \pm 9.6 \%$ in mild coronary artery disease group. $^{8}$

According to vessel score, the mean NLR was increased with the increase in number of vessel involved and this association was statistically significant $(\mathrm{p}=0.001)$.

According to Gensini score CAD was mild in 9.1\% (5) patients in group I and $23.6 \%$ (13) patients in group II and mild CAD was significantly low in group I $(p=0.04)$. Moderate CAD was found in $60 \%$ (33) patients in group É and 69.1\% (38) patients in group ÉÉ with insignificant difference $(p=0.31)$. Severe CAD was found in $30.9 \%$ (17) patients in group I and $7.3 \%$ (4) patients in group II and was significantly higher in group I $(p=0.001)$. So coronary artery disease severity was increased with increase in NLR.

Mean Gensini score was $42.3 \pm 24.9$ and $25.65 \pm 17.9$ in group-I and group-II respectively and the difference of mean Gensini score was statistically significant $(\mathrm{p}=0.001)$. In a related study by Zhang, et al. found mean Gensini score was $45.7 \pm 21.4$ in higher NLR group and 27.8 \pm 15.6 in lower NLR group and the difference was statistically significant $(p=<0.001) .{ }^{6}$ So our findings are consistent with existing studies.

In our study correlation co-efficient between NLR and vessel score was $r=0.30(p=0.004)$ and correlation co-efficient between NLR and Gensini score was $r=0.65(p=0.001)$. In a similar study conducted by Kaya, et al. the significant relationship between NLR and Gensini score was reported $(\mathrm{r}=0.422, \mathrm{p}=<0.001) .{ }^{7} \mathrm{We}$ found a moderate positive correlation between NLR and 
coronary artery disease severity in terms of Gensini score. With the increase of NLR, vessel score and Gensini score increased, indicating more severe coronary artery disease.

In this study univariate logistic regression analysis of variables likely to cause severe CAD revealed that age $>50$ years $(\mathrm{P}=0.03)$, hypertension $(\mathrm{P}=0.007)$, dyslipidemia $(\mathrm{P}=0.003)$, serum creatinine $(\mathrm{P}=0.04)$, total WBC count $(\mathrm{P}=0.006)$ and NLR $(\mathrm{p}=0.003)$ were independent predictors of severe coronary artery disease with odds ratio (OR) being 1.88, 3.93, 5.01, 5.54, 4.05 and 5.70, respectively. However, smoking $(p=0.38)$, positive family history of premature CAD $(p=0.44)$, diabetes mellitus $(p=0.107)$, neutrophil count $(p=0.09)$, lymphocyte count (0.14) and LVEF were not independent predictors of severe CAD. In multivariate logistic regression analysis, after adjustment of factors, age $>50$ years, hypertension, dyslipidemia and NLR were found the independent predictors of severe CAD with ORs being 3.27, 3.71, 4.95, and 5.73 respectively.

\section{Conclusion:}

From this study it may be concluded that increased neutrophil to lymphocyte ratio is associated with angiographically severe coronary artery disease in chronic stable angina patients and this association is independent of conventional cardiovascular risk factors.

Conflict of Interest - None.

\section{References:}

1. Montalescot G, Sechtem U, Achenbach S, Andreotti F, Arden C, Budaj A, et al. 2013 ESC guidelines on the management of stable coronary artery disease: the Task Force on the management of stable coronary artery disease of the European Society of Cardiology. Eur Heart J 2013; 34: 2949-3003.

2. Gibson PH, Cuthbertson BH, Croal BL, Rae D, El-Shafei $\mathrm{H}$, Gibson G, et al. Usefulness of neutrophil/lymphocyte ratio as predictor of new-onset atrial fibrillation after coronary artery bypass grafting. Am J Cardiol 2010; 105:186-191.

3. Tamhane UU, Aneja S, Montgomery D, Rogers EK, Eagle KA, Gurm HS. Association Between Admission Neutrophil to Lymphocyte Ratio and Outcomes in Patients With Acute Coronary Syndrome. Am J Cardiol 2008; 102: 653-657.

4. Nunez J, Nunez E, Bodi V, Sanchis J, Minana G, Mainar $\mathrm{L}$, et al. Usefulness of the neutrophil to lymphocyte ratio in predicting long-term mortality in ST segment elevation myocardial infarction. Am J Cardiol 2008; 101: 747-752.

5. Gensini GG. A more meaningful scoring system for determining the severity of coronary heart disease. Am J Cardiol 1983; 51: 606.

6. Zhang GY, Chen M, Yu ZM, Wang XD, Wang ZQ. Relation between neutrophil-to-lymphocyte ratio and severity of coronary artery stenosis. Genetic and Molecular Research 2014; 13: 9382-9389.

7. Kaya H, Ertas F, Islamoglu Y, Kaya Z, Atilgan ZA, Cil H, et al. Association between neutrophil to lymphocyte ratio and severity of coronary artery disease. Clin Appl Thromb Hemost 2014;20:50-54.

8. Na KY, Kim CW, Song YR, Chin HJ, Chae D-W. The association between kidney function, coronary artery disease, and clinical outcome in patients undergoing coronary angiography. J Korean Med Sci 2009; 24 Suppl:S87-594. 